\title{
Idiopathic CD4+T cell lymphocytopenia: A rare immunodeficiency disorder
}

Prabhas Prasun Giri ${ }^{1}$, Priyankar Pal ${ }^{1}$

Sri Lanka Journal of Child Health, 2014; 43(1): 59-60

(Key words: Idiopathic CD4+T cell lymphocytopenia; immune deficiency; opportunistic infections)

\section{Introduction}

Idiopathic CD4+T cell lymphocytopenia (ICL), a rare immunodeficiency disorder, is a heterogeneous clinical syndrome with the universal finding of persistent CD4+ T-lymphocyte depletion, in the absence of human immunodeficiency virus (HIV) 1 or 2 infection or other known causes of immunodeficiency $^{1,2}$. It was first described by Centers for Disease Control (CDC) in 1992 in individuals who suffered from recurrent opportunistic infections and persistently low CD4 counts but HIV serology was repetitively negative. Most of the ICL patients originally reported were adults; however the disease has subsequently been described in a small number of children and adolescents. The patients usually present with recurrent episodes of opportunistic infections like mycobacteria, candida, bacterial pneumonia and disseminated viral infections. We report a boy who presented with features compatible with ICL.

\section{Case report}

A 4 year 6 month old boy presented with recurrent episodes of cough, fever, loose stools, skin infections, ear discharge and oral thrush since the age of one month and history of four hospitalisations. At the age of one month he suffered from bacterial pneumonia, at 22 months post measles bronchopneumonia and at 3 years he had fever and convulsions with obtundation, followed by right sided hemiparesis. There was no history of intake of any immunosuppressants or immune-modifying drugs. Family members were healthy.

Examination revealed a poorly built and malnourished child (weight and height $<3^{\text {rd }}$ centile). The BCG scar was absent; skin was rough with multiple areas of post inflammatory hyperpigmentation and features of atopic dermatitis. There were many itchy lesions throughout the body, onychomycosis, right sided corneal opacity (Figure 1), oral thrush, an unusual blackish pigmentation of oral mucosa with cheilitis

${ }^{1}$ Institute of Child Health, Kolkata 17, India

(Received on 11 April 2013: Accepted after revision on 17 May 2013) and angular stomatitis. Liver was palpable $8 \mathrm{~cm}$ below right costal margin, firm in feel. There was bilateral chronic suppurative otitis media (CSOM), bilateral crepitations in the chest and residual paresis of right half of the body.

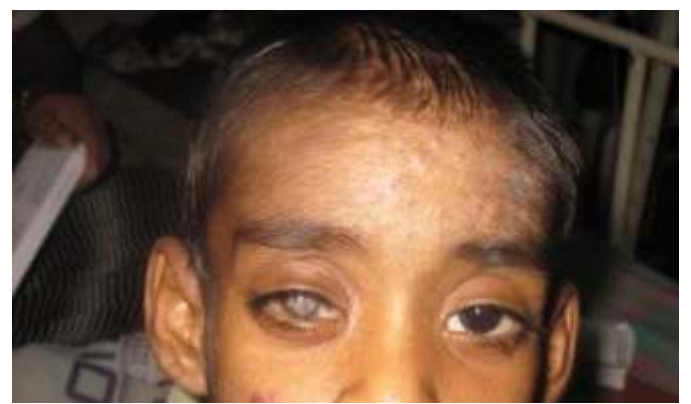

Figure 1: Right corneal opacity

*Permission given by parents to publish photograph

Investigations revealed mild anaemia (haemoglobin: 9.5g/dl), normal leucocyte, lymphocyte and platelet counts and eosinophilia (absolute eosinophil count 1,584). Immunological evaluation showed high immunoglobulin G, immunoglobulin A and immunoglobulin $\mathrm{E}$ ( $\mathrm{IgG}$ : 2,755, IgA: 431 IgE: 1,872) with a normal immunoglobulin $M$ level. CD4 count was 279 cells/microlitre (N: 500-2400), CD4\% was $9.39 \%$ (N: 23-48), CD8 count was 523 cells/microlitre (N: 300-1600), CD8\% was 17 (N: 14-33), CD4/CD8 was $0.53(\mathrm{~N}: 0.9-2.9)$. Thus there was $\mathrm{CD} 4$ lymphocytopenia with normal CD8 levels and consequent reversal of their ratio. CD4 \& CD8 counts repeated after 2 months showed persistently depressed levels of CD4 and the altered CD4/CD8 ratio (CD4 count was 272cells/microlitre). HIV 1 \& 2 were negative on 3 occasions. Tuberculin test was negative. Candida spp. were isolated from nail scrapings and liver biopsy revealed nonspecific inflammatory changes. Chest x-ray showed increased bronchovascular markings.

Bronchoalveolar lavage revealed M. tuberculosis. He was started on 4 drugs, isoniazid, rifampicin, pyrazinamide and ethambutol (HRZE) and the fever subsided after a few days. Owing to the disseminated nature and immunodeficiency, the plan of anti-TB drugs (ATD) was made as 
2HRZE+10HR. On follow up over the next 12 months he remained apparently well though intermittent breakthrough fever occurred.

\section{Discussion}

Idiopathic CD4 $\mathrm{T}$ cell lymphocytopenia (ICL) is a very rare immunodeficiency disorder with fewer than 50 paediatric cases reported throughout the world ${ }^{2}$. The definition of ICL in children includes the following criteria ${ }^{2}$ :

1. A CD4+T cell count of less than 1000 cells per cu $\mathrm{mm}$ in children 0 to 23 months of age and of less than 300 cells per cu $\mathrm{mm}$ in children 2 to 12 years of age, or a CD4+T lymphocyte count that is less than $20 \%$ of total lymphocytes on at least two separate measurements.

2. No serological evidence of infection on HIV testing (even if the child's mother is HIV seropositive).

3. Absence of any defined immunodeficiency or therapy associated with $\mathrm{T}$ cell depletion.

Our index case had a CD4 cell count less than 300 on 2 different occasions with absence of HIV seropositivity.

The aetiology of ICL is currently unknown. Research has focused on identifying abnormalities in various aspects of immune function and the role of any possible triggering viral agents ${ }^{3}$.

The mechanism of CD4 cell loss in ICL appears to be due to spontaneous and accelerated CD4+ cell apoptosis (programmed cell death) in patients with advanced immune suppression and opportunistic infections ${ }^{4}$. Recent views on potential mechanism for CD4 depletion include overexpression of Fas / Fas ligand leading to apoptosis ${ }^{5}$, defective cytokine production, regenerative failure of haematopoietic stem cells, impaired thymic $\mathrm{T}$ cell maturation and autoantibody against CD4 cells.

Opportunistic infections occur when the absolute CD4 cell count drops below 200 cells/cu mm. Conditions most commonly associated are mycobacterial infections, fungal infections (candida, cryptococcus, aspergillus, histoplasma) and viral infections (human papilloma virus, varicella, cytomegalovirus, Epstein-Barr virus, John Cunningham virus). Our index case suffered from mycobacterial and fungal infections. Recurrent bacterial infections are rare but are seen in children. Allergic conditions such as atopic dermatitis have often been linked to $\mathrm{ICL}^{6}$.
Aside from persistently low CD4+ T cell counts, laboratory findings in ICL are variable.

There is no standard treatment for ICL, except for management of the associated conditions and the prompt treatment of infections. Emphasis should be on prevention of infection with antibiotic and antifungal prophylaxis. Some novel approaches like allogenic bone marrow transplantation, IL-2 administration have been tried in adult patients, but their use in children is limited.

\section{References}

1. Unexplained CD4+ T-lymphocyte depletion in persons without evident HIV infection--United States. MMWR Morbidity and Mortality Weekly Reports 1992; 41:541.

2. Smith, DK, Neal JJ, Holmberg SD. Unexplained opportunistic infections and CD4+ T-lymphocytopenia without HIV infection: An investigation of cases in the United States. The Centers for Disease Control Idiopathic CD4+ T-lymphocytopenia Task Force. New England Journal of Medicine 1993; 328:373. http://dx.doi.org/10.1056/NEJM199302113280 601

3. Menon BS, Shuaib IL, Zamari M, et al. Idiopathic CD4+ T-lymphocytopenia in a child with disseminated cryptococcosis. Annals of Tropical Paediatrics 1998; 18:45.

4. Garry RF, Fermin CD, Kohler PF, et al. Antibodies against retroviral proteins and nuclear antigens in a subset of idiopathic CD4+ $\mathrm{T}$ lymphocytopenia patients. AIDS Research and Human Retroviruses 1996; 12:931.

http://dx.doi.org/10.1089/aid.1996.12.931

5. Laurence J, Mitra D, Steiner $M$, et al. Apoptotic depletion of CD4+ $\mathrm{T}$ cells in idiopathic CD4+ T lymphocytopenia. Journal of Clinical Investigation 1996; 97:672. http://dx.doi.org/10.1172/JCI118464

6. Hardman CM, Baker BS, Lortan J, et al. Active psoriasis and profound CD4+ lymphocytopenia. British Journal of Dermatology 1997; 136:930. http://dx.doi.org/10.1046/j.13652133.1997.018 02.x 